\title{
Confronting the EPOS-LHC model predictions on the charged particle and muon attenuation lengths of EAS with the measurements of the KASCADE-Grande observatory
}

\author{
J.C. Arteaga-Velázquez ${ }^{1, a, b}$, W.D. Apel ${ }^{2}$, K. Bekk², M. Bertaina ${ }^{3}$, J. Blümer ${ }^{2,4}$, H. Bozdog ${ }^{2}$, I.M. Brancus ${ }^{5}$, \\ E. Cantoni ${ }^{3,6, c}$, A. Chiavassa ${ }^{3}$, F. Cossavella ${ }^{4, d}$, K. Daumiller ${ }^{2}$, V. de Souza ${ }^{7}$, F. Di Pierro ${ }^{3}$, P. Doll ${ }^{2}$, R. Engel ${ }^{2}$, \\ J. Engler ${ }^{2}$, B. Fuchs ${ }^{4}$, D. Fuhrmann ${ }^{8, e}$, A. Gherghel-Lascu ${ }^{5}$, H.J. Gils ${ }^{2}$, R. Glasstetter ${ }^{8}$, C. Grupen ${ }^{9}$, A. Haungs ${ }^{2}$, D. Heck ${ }^{2}$, \\ J.R. Hörandel ${ }^{10}$, D. Huber ${ }^{4}$, T. Huege ${ }^{2}$, K.-H. Kampert ${ }^{8}$, D. Kang ${ }^{4}$, H.O. Klages ${ }^{2}$, K. Link ${ }^{4}$, P. Łuczak $^{11}$, H.J. Mathes ${ }^{2}$, \\ H.J. Mayer² ${ }^{2}$ J. Milke², B. Mitrica ${ }^{5}$, C. Morello ${ }^{6}$, J. Oehlschläger ${ }^{2}$, S. Ostapchenko ${ }^{2, f}$, N. Palmieri ${ }^{4}$, M. Petcu ${ }^{5}$, T. Pierog ${ }^{2}$, \\ H. Rebel ${ }^{2}$, M. Roth ${ }^{2}$, H. Schieler ${ }^{2}$, S. Schoo ${ }^{2}$, F.G. Schröder ${ }^{2}$, O. Sima ${ }^{12}$, G. Toma ${ }^{5}$, G.C. Trinchero ${ }^{6}$, H. Ulrich ${ }^{2}$, \\ A. Weindl ${ }^{2}$, J. Wochele ${ }^{2}$, and J. Zabierowski ${ }^{11}$ \\ 1 Institute of Physics and Mathematics, Universidad Michoacana de San Nicolás de Hidalgo, Morelia, Mexico \\ 2 Institut für Kernphysik, KIT - Karlsruher Institut für Technologie, Germany \\ 3 Department of Physics, University of Torino and INFN, Torino, Italy \\ 4 Institut für Experimentelle Kernphysik, KIT - Karlsruher Institut für Technologie, Germany \\ 5 Horia Hulubei National Institute of Physics and Nuclear Engineering, Bucharest, Romania \\ 6 Osservatorio Astrofisico di Torino, INAF Torino, Italy \\ 7 Universidade São Paulo, Instituto de Física de São Carlos, Brasil \\ 8 Fachbereich Physik, Universität Wuppertal, Germany \\ 9 Department of Physics, Siegen University, Germany \\ 10 Department of Astrophysics, Radboud University Nijmegen, The Netherlands \\ 11 National Centre for Nuclear Research, Department of Astrophysics, Lodz, Poland \\ 12 Department of Physics, University of Bucharest, Bucharest, Romania
}

\begin{abstract}
KASCADE-Grande was an air-shower experiment designed to study cosmic rays between $10^{16}$ and $10^{18} \mathrm{eV}$. The instrument was located at the site of the Karlsruhe Institute of Technology, Germany at an altitude of $110 \mathrm{~m}$ a.s.l. and covered an area of $0.5 \mathrm{~km}^{2}$. KASCADE-Grande consisted of several detector systems dedicated to measure different components of the EAS generated by the primary cosmic rays, i.e., the muon and the electron contents of the air-shower. With such a number of EAS observables and the precision of the measurements, the KASCADE-Grande data can be used to not only study in detail the properties of cosmic rays but also to test the predictions of hadronic-interaction models. In this work, in particular, the attenuation lengths of the muon number and the charged number of particles of EAS in the atmosphere were extracted from the KASCADE-Grande data and the results were compared with the predictions of the new EPOS-LHC hadronic-interaction model.
\end{abstract}

\section{Introduction}

An important step forward in the research of cosmic rays would be to count with a theoretical tool to find out the main characteristics of the primary cosmic rays of the highest energies from the measurements of its corresponding extensive air shower (EAS) at ground. Such a tool is still missing, however, major advances have been performed recently [1-3]. These improvements have been triggered by the arrival of fresh data on hadronic interactions from both the first runs at the

\footnotetext{
a e-mail: arteaga@ifm.umich.mx

${ }^{\mathrm{b}}$ Corresponding author

${ }^{\mathrm{c}}$ Now at: Istituto Nazionale di Ricerca Metrologica, INRIM,

Torino, Italy

${ }^{\mathrm{d}}$ Now at: DLR Oberpfaffenhofen, Germany

e Now at: University of Duisburg-Essen, Duisburg, Germany

${ }^{\mathrm{f}}$ Now at: University of Trondheim, Norway
}

Large Hadron Collider (LHC) and from modern forward physics experiments (such as the LHCf [4], CASTOR [5], TOTEM [6] and NA61/SHINE [7]), which have been used to calibrate the new hadronic interaction models. Interestingly, part of these data has been collected up to energies of $\sqrt{s_{p p}} \approx 8 \mathrm{TeV}$, which is equivalent to a collision of a $3.2 \times 10^{16} \mathrm{eV}$ proton with a target nucleon at rest. This energy is well above the location of the knee feature $\left(\approx 10^{15} \mathrm{eV}\right.$ at the laboratory system) presented by the spectrum of cosmic rays.

In spite of these advances, however, there are still theoretical uncertainties $[3,8]$ that hampered the determination of the energy and the exact composition of cosmic rays at energies above $10^{15} \mathrm{eV}[9,10]$. Therefore, complementary studies are needed to find new clues to guide the construction of hadronic interaction models.

Such efforts are currently in progress in several EAS observatories around the world, e.g., the

This is an Open Access article distributed under the terms of the Creative Commons Attribution License 4.0, which permits unrestricted use, distribution, and reproduction in any medium, provided the original work is properly cited. 
KASCADE-Grande experiment [11] and the Pierre Auger Observatory [10], where the employment of complementary and different instrumental techniques are an important requisite for this kind of studies.

In this paper, we will present a particular analysis performed with the KASCADE-Grande data to test a postLHC hadronic interaction model called EPOS-LHC [2]. We will find out how the recent improvements experienced by the EPOS model have affected its predictions on the muon and charged particle attenuation lengths in cosmicray induced air showers and on the zenith angle evolution of the EAS muon content by comparing the expectations from the models with the data of the KASCADE-Grande observatory. But, before turning our attention to the main topic of the present contribution, we will present a brief overview of the KASCADE-Grande experiment and some of its principal achievements during the last years.

\section{The KASCADE-Grande experiment}

In spite of the enormous experimental efforts and theoretical developments in the field of cosmic rays, nowadays there is still no definite answers to several fundamental questions about the origin, the nature, the acceleration processes and the propagation in space of these energetic particles.

Certainly, the resolution of these enigmas will require, on the one hand, more theoretical work (maybe fresh ideas) along with more precise measurements of highenergy cosmic rays, and in the other hand, complementary observations of the universe with different astronomical windows at the high-energy regime, namely neutrinos and $\gamma$-rays. In speaking here concerning precise cosmic ray experiments, we can talk about the KASCADEGrande observatory, which has provided important keys for understanding the cosmic ray energy regime between $10^{15}$ and $10^{18} \mathrm{eV}$.

The KASCADE-Grande experiment was an air-shower ground-based detector, which was located until 2013 in Germany at the Karlsruhe Institute of Technology at an altitude of $110 \mathrm{~m}$ a.s.l. [11]. It was dedicated to investigate cosmic ray particles within the energy interval of $1 \mathrm{PeV}$ and $1 \mathrm{EeV}$, which is characterized by an enigmatic bending around $10^{15} \mathrm{eV}$ in the corresponding spectrum known as the knee. At KASCADE-Grande the energy, composition and arrival directions of cosmic rays were studied indirectly by measuring the properties of airshowers induced by the former in the atmosphere.

The KASCADE-Grande observatory covered an effective area for EAS detection of $0.5 \mathrm{~km}^{2}$. The instrument consisted of different detection systems and was designed to measure and separate several components of air-showers, e.g. the muon content and the total charged particle number ${ }^{1}$ [11]. Measurements of the charged number of particles were accomplished with a $0.5 \mathrm{~km}^{2}$ surface detector named Grande, which was composed of an array of 37 plastic scintillator detectors with a spacing of $\approx 137 \mathrm{~m}$. Each station had a sensitive area of $10 \mathrm{~m}^{2}$ and a threshold energy of $3 \mathrm{MeV}$ for normal incident

\footnotetext{
${ }^{1}$ Defined as the sum of the number of muons and electrons in the EAS.
}

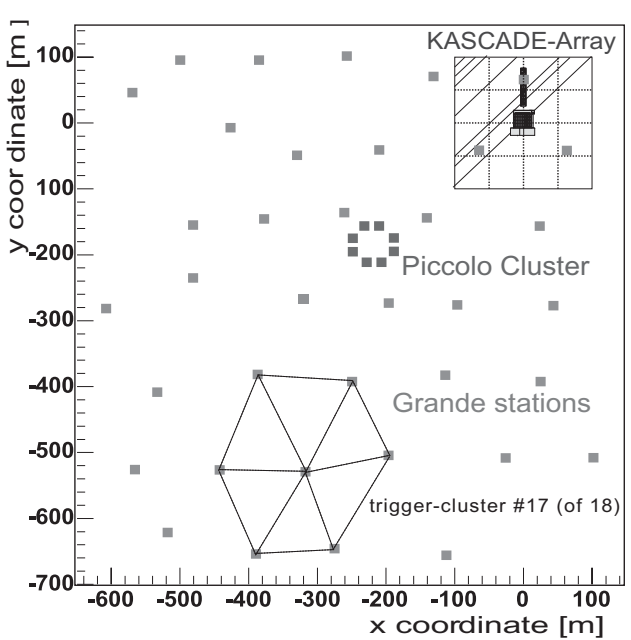

Figure 1. The KASCADE-Grande layout. Different detection systems are shown: the Grande and the KASCADE arrays, the central detector (CD), the Piccolo cluster and the Muon Tracking Detector (MTD).

particles. On the other hand, muon measurements were performed with the array of 192 shielded detectors of the original KASCADE observatory [12]. These detectors had a smaller separation $(\approx 13 \mathrm{~m})$ and were part of the 12 outer clusters of the $200 \times 200 \mathrm{~m}^{2}$ KASCADE array. The total sensitive area of the $\mu$-detectors was about $622 \mathrm{~m}^{2}$ and the threshold energy for vertical incidence, $230 \mathrm{MeV}$. Several other instruments of the KASCADE-Grande instrument are described elsewhere $[11,13]$, but since they are not relevant for our study, they will not be described here. The layout of the KASCADE-Grande experiment is shown in Fig. 1.

As regards the performance of the instrument, it was studied in [11]. The angular resolution of the Grande detector was $\approx 0.7^{\circ}$, while the $N_{c h}$ precision and the core location accuracy were $\leq 15 \%$ and $\approx 5 \mathrm{~m}$, respectively. The systematic errors were obtained independently of the simulations for a subset of data recorded by both the KASCADE and the Grande detectors. The uncertainty calculations were done by comparing event-by-event the values of the shower parameters estimated on the one hand with the Grande data and, on the other, with the KASCADE measurements. Muon systematics, for KASCADE-Grande, were found to be lower than $25 \%$. The latter were calculated from MC simulations, as there was only one muon array available in the KASCADEGrande facility.

\subsection{Energy and mass composition studies with the KASCADE-Grande facility}

Several astrophysical models predict the existence of individual kinks in the energy spectra of cosmic ray particles with definite chemical composition. The kink position could scale with the proton number $(Z)$ [14] of the cosmic nuclei or the corresponding atomic mass $(A)$ [15]. Details depend upon the specific acceleration model and the propagation mechanisms.

First evidence in favor of this scenario was presented by the KASCADE Collaboration, which by an unfolding 
technique of the data on the $N_{e}$ and $N_{\mu}$ contents of EAS registered with the KASCADE detector around the knee, obtained the energy spectra for five representative mass groups: $\mathrm{H}, \mathrm{He}, \mathrm{C}, \mathrm{Si}$ and $\mathrm{Fe}$, in the framework of the QGSJET 01 and SIBYLL 2.1 high-energy hadronic interaction models [16].

The unfolded results showed, in fact, the presence of individual knees in the energy spectra of the light, the intermediate and Si mass groups at energies between $10^{15}$ and $10^{17} \mathrm{eV}$, whose corresponding positions depend on the composition of the mass groups. These conclusions were proved to be independent of the high-energy hadronic interaction model used to interpret the data. Although, the relative abundances of the mass group spectra were observed to be sensitive to the hadronic interaction model used in the analysis. Interestingly, the unfolding analysis also showed that the structure known as the knee in the allparticle energy spectrum is the result of the behavior of the light and intermediate mass groups.

The uncertainties of the measurements, however, did not allow to answer the question whether the positions of the knees in the energy spectra of the elemental mass groups depend on the electric charge or the atomic mass of the primary nuclei. It was soon recognized that measurements of the iron component could help to elucidate the question. In particular, a change towards a heavy composition in the energy spectrum of cosmic rays was expected above $\mathrm{PeV}$ energies in conventional acceleration models, a picture that was consistent with KASCADE data [16]. Besides, it was also expected the presence of an iron knee, at energies $\gtrsim 10^{17} \mathrm{eV}$, which were beyond the reach of the KASCADE experiment. The issue was investigated with the KASCADE-Grande observatory, which extended the KASCADE measurements up to energies of $10^{18} \mathrm{eV}$ [11].

The KASCADE-Grande results on the analyses of elemental composition of cosmic rays in the range from $10^{16}$ to $10^{18} \mathrm{eV}$ were presented in $[17,18]$ and [19]. In the analyses of $[17,18]$ cosmic ray data was divided into two mass groups on an event-by-event basis according to a cut on an energy dependent parameter, which is defined terms of the muon content and the number of charged particles of the EAS. The cut was guided by MC simulations. Then, after separation, an energy calibration procedure was applied on the data of each mass group depending on the composition and arrival zenith angle of the event, using relations derived from MC simulations. As a final result, the energy spectra for the light and heavy mass groups were obtained. Interestingly, the heavy component resulted to be the dominant mass group around $10^{17} \mathrm{eV}$. Even more, at this energy, the existence of a knee-feature in the heavy mass spectrum was discovered. The presence of a heavy knee in the cosmic ray data was later shown to be independent of the high-energy hadronic interaction model [20]. On the contrary, the position of this kink was found to depend on the hadronic model [20].

An unexpected finding was discovered, on the other hand, in the spectrum of the light component, namely a striking recovery that leads to an ankle-feature a little bit above $10^{17} \mathrm{eV}$ [18]. Might it be the first clue of the expected galactic-extragalactic transition in the cosmic ray spectrum, the next generation of cosmic ray observatories may solve the question?

The existence of the iron knee came finally from a more sophisticated analysis performed on the KASCADEGrande data in [19]. The method consisted of applying the KASCADE unfolding technique on the measured bidimensional distribution of the number of charged particles and the muon content of air showers to reconstruct the spectra for five elemental primaries representing the chemical composition of cosmic rays using QGSJET II-02. The unfolding results of KASCADEGrande were shown to be all consistent with the spectra at lower energies obtained from the KASCADE data employing also the same hadronic interaction model [19]. Regarding the position of the iron knee, it was found at around $80 \mathrm{PeV}$, which is correlated with the location of the heavy knee discussed above. Such a position of the iron knee is therefore compatible with rigidity dependent scenarios, assuming that the $\mathrm{PeV}$ knee is caused by a decrease in the flux of light primaries, i.e., protons and/or helium [19].

The composition analysis stage in KASCADE-Grande has not yet finished, as different reconstruction techniques are still being studied with the full statistics accumulated during the lifetime of the experiment. One of them, which is in progress, will combine the KASCADE and the KASCADE-Grande data to reconstruct a single cosmic ray spectrum in the energy range from $10^{15} \mathrm{eV}$ to $10^{18} \mathrm{eV}$.

\subsection{Tests of hadronic interaction models at the KASCADE-Grande observatory}

An important limitation to the composition and energy analyses performed on KASCADE-Grande data has been the role of the hadronic interaction models at high energies. The impact upon the KASCADE-Grande main results has been long investigated [20]. But, in addition, the test of high-energy hadronic interaction models used for the reconstruction of the results has been also an active topic of research with the KASCADE-Grande facility. Some of the newest analyses will be reported in these proceedings $[21,22]$.

One of the latest analysis reveals a serious discrepancy between the predicted and measured density of charged particles at $500 \mathrm{~m}$ from the EAS core at the shower plane [21]. According to simulations, this observable named as $S(500)$ is ideal to reconstruct the all-particle energy spectrum of cosmic rays as it is composition independent. However, the differences between the expectations based on QGSJET II-02 and the measurements on $S(500)$ lead to a total energy spectrum which is not in agreement with the one reconstructed from the previous analyses based on the $N_{\mu}$ and the $N_{c h}$ numbers for the same hadronic interaction model.

Another study was also recently performed on the muon pseudorapidity of EAS measured with the Muon Tracking Detector (MTD) of the KASCADEGrande facility [22]. This observable is correlated with the longitudinal development of air showers, while its distribution, with the mean logarithmic mass, $\langle\ln A\rangle$, of cosmic rays. The correlation is of course model dependent. The study has shown the differences on the results of $\langle\ln A\rangle$ 
when data is interpreted with several high-energy hadronic interaction models: QGSJET II-02, EPOS 1.99 and the newest versions QGSJET II-04 and EPOS-LHC, showing that the latter ones tend to assign to experimental data a higher value of mean logarithmic mass than the earlier versions.

More tests of high-energy hadronic interaction models are underway. For instance, a research program to study the muon content of air showers using the MTD and the KASCADE muon array has been designed in order to search for clues that could lead us to trace the origin of a major discrepancy between the predicted and the measured zenith-angle evolution of the $N_{\mu}$ content of EAS detected with the KASCADE-Grande observatory [23]. These predictions were estimated for the QGSJETII-02, QGSJETII-04, SIBYLL 2.1 and the EPOS 1.99 high-energy hadronic interaction models. In the present study, we will extend that research to the new EPOS-LHC model, and will hope to find out whether the referred post-LHC model can provide us with a better description of the KASCADE-Grande data on the effective attenuation of the muon number of air showers in the atmosphere at least at the energy range studied with the observatory $\left(E=10^{16}-10^{18} \mathrm{eV}\right)$.

Clues that the EPOS-LHC model has deficiencies at higher energies $\left(E \geq 10^{18} \mathrm{eV}\right)$ have been found recently by the Pierre Auger Collaboration [24]. They have reported that the EPOS-LHC model fails to describe the muon content and the muon production depth of air-showers [24], although, it gives a better description of the data than other models at ultra high-energies, as to the muon number in EAS. Whether it happens or not to be also the case at lower energies $\left(E=10^{16}-10^{17} \mathrm{eV}\right)$ is one of the questions that will be addressed with the present preliminary investigation using the KASCADE-Grande data.

\section{EPOS-LHC}

EPOS-LHC is one of the latest post-LHC high-energy hadronic interaction models, which has been tuned to reproduce several experimental data on hadronic interactions including results of different LHC experiments up to $\sqrt{s}=7 \mathrm{TeV}[2,3]$. Like its predecessor, the EPOS 1.99 model, EPOS-LHC is based on the theory of the Gribov-Regge multiple scattering, perturbative QCD and string fragmentation. However, EPOS-LHC includes some changes regarding the flow parameterization of the high-density core of thermalized matter produced during $p-p$ or heavy-ion collisions, the decay of the core (which is now treated statistically) and the baryon formation (with multistrange production more important than string fragmentation) [2].

The update on the EPOS model involves also modifications of hadron production parameters that are important for the EAS development, for instance, of the proton-proton scattering total cross section, $\sigma_{p p}^{T o t}$, the multiplicity of secondary particles at high-energies and the (anti)baryon production [3]. An important parameter for the depth of shower maximum is $\sigma_{p p}^{T o t}$. EPOS-LHC and EPOS 1.99 predict almost similar values of the cross section below $\sqrt{s}=7 \mathrm{TeV}$. However, the predictions

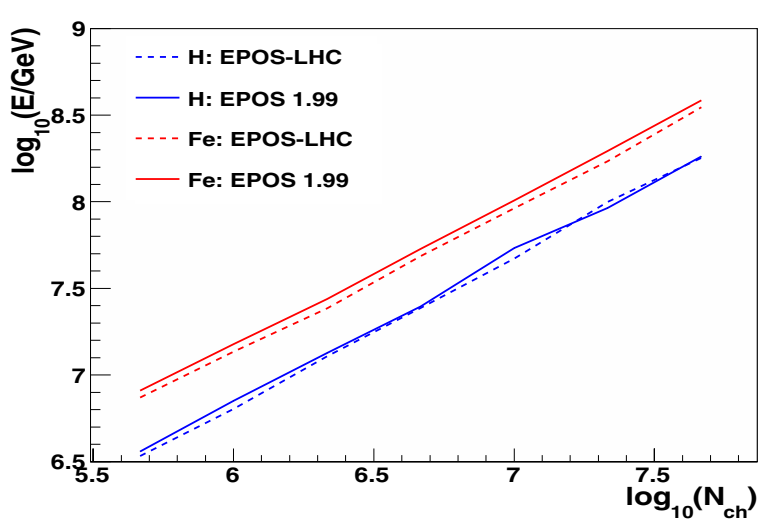

Figure 2. Predictions of EPOS-LHC and EPOS 1.99 for the dependence of the primary energy on $N_{c h}$ for two different primary compositions at the site of the KASCADEGrande observatory for the respective experimental threshold for secondary particles. No experimental effects on the EAS observables were taken into account. Only showers with $\theta \leq 18^{\circ}$ were considered.

begin to separate at higher energies. In particular, it is found that the total cross section increases faster in EPOS-LHC than in the EPOS 1.99 model. On the other hand, the averaged multiplicity for $\pi$-air collisions at high energies is now bigger in EPOS-LHC than for its pre-LHC version. The multiplicity distributions have also changed, being more reliable for the new EPOS version, at least up to $\sqrt{s}=7 \mathrm{TeV}$, due to calibration with the CMS and ALICE data. Important for the predictions of the muon content of air showers and the charged number of particles are the modifications in the (anti) baryon production and its spectrum. In EPOS-LHC, the forward (anti)baryon production turns to be more important at high energies than for EPOS 1.99. However, the number of muons is very similar in both models. On the contrary, the charged number of particles is modified. EPOS-LHC predicts higher $N_{c h}$ values than EPOS 1.99. For KASCADE-Grande and vertical showers $\left(\theta \leq 18^{\circ}\right)$ differences between the two models for primary energies $\geq 10^{15.5} \mathrm{eV}$ can reach up to $5 \%$ and $13 \%$ in case of the $N_{\mu}$ and $N_{c h}$ observables respectively (for the latter see Fig. 2). As regards the $\log _{10}\left(N_{\mu}\right) / \log _{10}\left(N_{c h}\right)$ ratio, mean differences are smaller than $0.85 \%$ (cf. Fig. 3). An important difference between the two models is found for the energy spectrum of muons at ground level, as EPOSLHC predicts a harder spectrum. This difference could be very important for the investigation that we are performing as it affects the zenith-angle evolution of the muon content of EAS in the atmosphere.

\section{Description of the tests}

The goal will be to test the EPOS-LHC model by studying the behavior of the $N_{\mu}$ observable with the zenith angle of the showers and our key parameter will be the muon attenuation length, as extracted with the Constant Intensity Cut method (CIC). The strategy is to compare the measured value for this parameter with the predictions from the model for protons and iron nuclei as extreme 


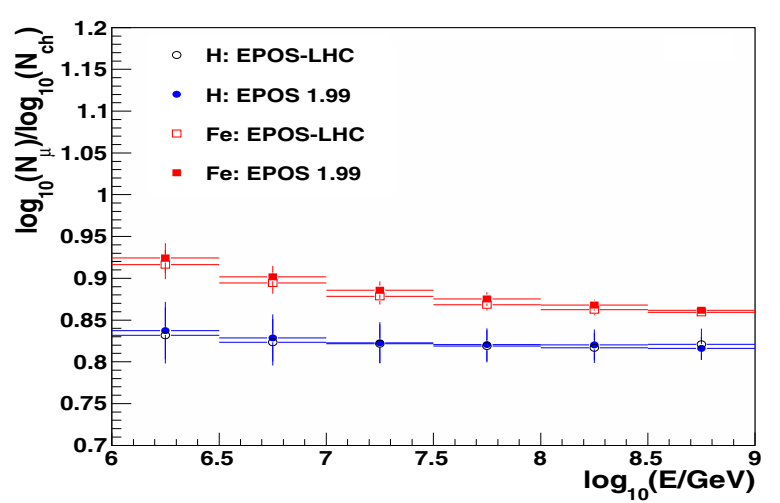

Figure 3. Predictions of EPOS-LHC and EPOS 1.99 for the energy dependence of the ratio $\log _{10}\left(N_{\mu}\right) / \log _{10}\left(N_{c h}\right)$ for EAS generated for different primary compositions. No experimental effects on the EAS observables were taken into account. Only EAS with $\theta \leq 18^{\circ}$ were considered.

composition assumptions, and look if the experimental value lies between these extreme values.

The above technique will also be applied with the $N_{c h}$ data in order to test the predictions of EPOS-LHC on the respective attenuation length.

\section{The experimental and $\mathrm{MC}$ data}

For the analysis, we have selected a special data set to reduce as much as possible the systematic uncertainties affecting our main observables, i.e, the number of muons and charged particles in the EAS. In particular, we have chosen events detected only inside a central fiducial area of the Grande detector to avoid border and punch-through effects and to reduce the muon systematic errors, which have a dependence on the distance of the shower core to the center of the KASCADE muon clusters. On the other hand, we consider showers with arrival zenith-angles below $40^{\circ}$ and a muon size $\log _{10}\left(N_{\mu}\right)>4.6$. Besides, we restrict ourselves to events recorded during stable runs with all KASCADE muon clusters active at the time of detection and data that were successfully reconstructed with the KASCADE-Grande algorithms. After applying the selection criteria, the experimental data set consisted of 2, 744, 950 showers, all of them recorded during the DAQ period from December 2003 to October 2011.

On the other hand, MC simulations were generated with the EPOS-LHC and the Fluka v2011.2b models for the high- and the low-energy $(E \leq 200 \mathrm{GeV})$ hadronic interactions, respectively. CORSIKA v7.400 was employed to simulate the EAS development, while a GEANT 3.21 based code was used to simulate the complete response of the KASCADE-Grande detector. Both MC and experimental data were reconstructed with the standard KASCADE-Grande reconstruction software described in [11]. About $1.2836 \times 10^{6} \mathrm{MC}$ events were generated with energies between $10^{15}$ and $10^{18} \mathrm{ev}$ from a primary flux with spectral index $\gamma=-2.0$ and zenith angles in the interval $\left[0^{\circ}, 42^{\circ}\right]$. We prepared data sets for five representative primaries: protons, He, C, Si and Fe. All of them were also combined in equal proportions to get an additional data set for a mixed composition scenario. Data

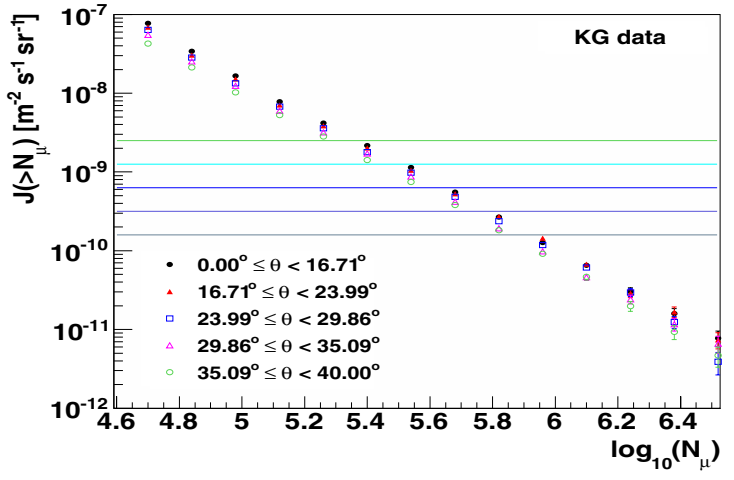

Figure 4. Integral muon fluxes for five zenith angle intervals derived from the measurements with the KASCADE-Grande observatory. The muon correction function was already applied to the data. The CIC cuts employed in this work are shown as horizontal lines. From below to above, the CIC cuts are: $\log _{10}\left[J_{\mu} / \mathrm{m}^{2} \cdot \mathrm{s} \cdot \mathrm{sr}\right]=-9.80,-9.50,-9.20,-8.90,-8.60$.

was also re-weighted to get different data sets for spectral indexes equivalent to $\gamma=-3.2,-3.0$ and -2.8 .

The efficiency and systematic uncertainties for the selected experimental data were investigated with MC simulations. According to EPOS-LHC, data has an energy threshold for maximum efficiency around $10^{16.0 \pm 0.1} \mathrm{eV}$, depending on the composition and the arrival zenith angle. As regards the systematic errors, mean values are smaller than $15 \%$ and $25 \%$ for $N_{c h}$ and $N_{\mu}$, respectively. To correct the muon number of EAS for instrumental and reconstruction effects a correction function was employed (for more details, see references [23,25]) ${ }^{2}$. After corrections, EPOS-LHC predicts that $N_{\mu}$ has a mean systematic error $<5 \%$ in the region of maximum efficiency.

\section{Estimation of $\Lambda_{\mu}$}

In order to calculate the muon attenuation length of muons in the atmosphere, we apply the standard CIC method as described in [23]. First we reconstruct the integral muon spectra, $J_{\mu}\left(>N_{\mu}, \theta\right)$ for different zenith angle intervals, which are selected in such a way that they have the same acceptance. For MC data and due to the lack of an energy normalization scale, we have normalized the simulated flux in such a way that the corresponding integral flux for the first bin of zenith angle $\left(\Delta \theta=\left[0^{\circ}, 16.71^{\circ}\right]\right)$ coincides with the experimental one inside the interval $\log _{10}\left(N_{\mu}\right)=$ $[5.1,5.6]$. Once the integral fluxes are estimated, we apply five CIC cuts in the region of maximum efficiency and statistics, in particular, within the interval $\log _{10}\left[\mathrm{~J}_{\mu} / \mathrm{m}^{2}\right.$. $\mathrm{s} \cdot \mathrm{sr}]=[-9.8,-8.6]$ (see Fig. 4). Then, for each cut, an attenuation curve $\log _{10}\left[N_{\mu}(\theta)\right]$ is constructed from the intersecting points between the line representing the CIC cut and the integral fluxes. Finally, the best $\Lambda_{\mu}$ value

\footnotetext{
2 The muon correction function is based on QGSJET II02 simulations and has a mild dependence on the hadronic interaction models. The function is calibrated with data sets derived for a mixed composition assumption and spectral index $\gamma=-3$. It has four parameters: the arrival direction of the EAS, the coordinates of the shower core position and the shower size.
} 
Table 1. Muon attenuation lengths extracted from Monte Carlo and experimental data. $\Lambda_{\mu}$ is presented along with its total error (statistical and systematic errors are added in quadrature).

\begin{tabular}{c|c|c|c|c|c|c}
\hline & QGSJETII & QGSJETII04 & EPOS 1.99 & SIBYLL 2.1 & EPOS-LHC & KG data \\
\hline$\Lambda_{\mu}\left(\mathbf{g} / \mathrm{cm}^{2}\right)$ & $705.95_{-105.36}^{+82.93}$ & $735.11_{-143.75}^{+74.38}$ & $561.71_{-59.20}^{+47.41}$ & $743.16_{-203.36}^{+44.35}$ & $823.16_{-148.46}^{+153.23}$ & $1255.63_{-193.59}^{+201.31}$ \\
\hline$\Lambda_{c h}\left(\mathbf{g} / \mathbf{c m}^{2}\right)$ & $193.63_{-21.26}^{+22.43}$ & $197.41_{-13.42}^{+10.67}$ & $180.08_{-21.84}^{+31.24}$ & $180.97_{-9.33}^{+17.21}$ & $217.56_{-13.12}^{+26.38}$ & $231.07_{-47.52}^{+17.36}$ \\
\hline
\end{tabular}

that describes the set of attenuation curves is extracted by performing a global fit (see Fig. 5) with the formula

$$
N_{\mu}(\theta)=N_{\mu}^{0} \exp \left[-X_{0} \sec (\theta) / \Lambda_{\mu}\right]
$$

where $X_{0}=1022 \mathrm{~g} / \mathrm{cm}^{2}$ is the mean atmospheric depth at the site of the KASCADE-Grande experiment and $N_{\mu}^{0}$ is a normalization parameter, which is also a fit parameter depending upon the CIC curve.

The value of the muon attenuation length calculated for EPOS-LHC in the setting of a mixed composition assumption with $\gamma=-3$ is shown in Table 1 . The errors include the statistical and systematic uncertainties added in quadrature. Systematic errors were only calculated for uncertainties in the primary composition and the spectral index. The errors in composition were obtained by repeating the calculation for a light $(\mathrm{H} \& \mathrm{He}$ on equal proportions) and a heavy mass group ( $\mathrm{Si} \& \mathrm{Fe}$ on equal abundances, respectively). We did not use single primaries because of the low statistics. For the case of the uncertainties in the spectral index, additional calculations were performed for $\gamma=-2.8$ and -3.2 . The result for $\Lambda_{\mu}$ as estimated from the experimental data is also presented in Table 1. The total error is obtained by summing in quadrature the statistical and systematic errors. Here, a more detailed calculation of the systematic uncertainties was performed. The estimation includes errors from the CIC method, the global fit, the systematic errors of the corrected muon size, the muon correction function and its mild hadronic model dependence as well as the EAS core position at ground. In Table 1, we have also included the predictions of other high-energy hadronic interaction models, namely QGSJETII-02, QGSJETII-04, EPOS 1.99, SIBYLL 2.1, as estimated in reference [23] along with their corresponding total errors.

From Table 1, we can observe a clear deviation of the predictions for $\Lambda_{\mu}$ from the measured value. Deviations vary between $1.75 \sigma$ and $3.48 \sigma$. The former is found for EPOS-LHC, and the latter for EPOS 1.99. The results suggest that, from all high-energy hadronic models, it is EPOS-LHC the one that provides the closest prediction to the measured $\Lambda_{\mu}$. By comparing the results from EPOSLHC and EPOS 1.99, we can see that the changes in the former have resulted in an improvement as regards its prediction for $\Lambda_{\mu}$

The calculation for EPOS-LHC should be considered as preliminary, as the estimation of the systematic errors is incomplete and the analysis is performed with a MC data sample of limited size. However, it is interesting to note that these preliminary results indicate that EPOS-LHC seems to describe better than the other hadronic interaction models the behavior of $N_{\mu}$ from air showers with the same energy at different zenith angles.

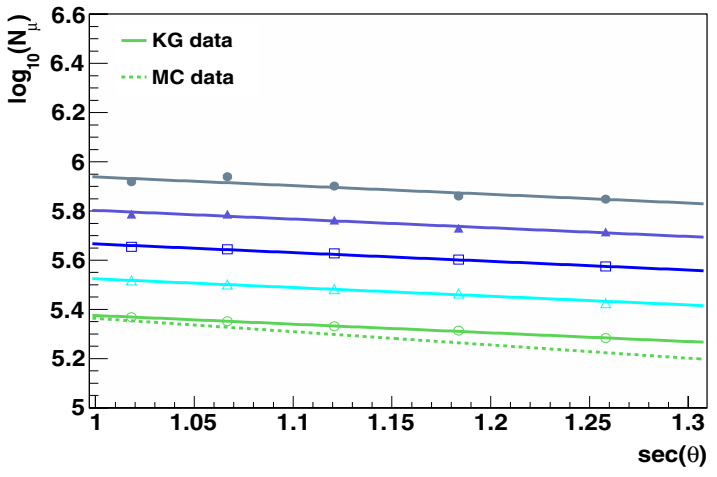

Figure 5. Muon attenuation curves extracted with the CIC method from the experimental KASCADE-Grande data. The result of the global fit with equation (1) is shown with solid lines. The highest curve correspond to the lowest CIC cut. The segmented line is an example of the result from the global fit applied to the EPOS-LHC data (mixed composition scenario with $\gamma=-3)$.

What does this result implies for the predictions of the MC models? Well, if at $\theta=0^{\circ}$, we normalize the expectations from MC models with the measured values for $N_{\mu}$, we will see an excess of muons in the experimental data over the predictions of MC models, which increases with the zenith angle. This discrepancy could be related with the muon excess observed by the Pierre Auger Collaboration in EAS at ultra-high energies.

\section{The calculation of $\Lambda_{c h}$}

We have also applied the method described in the last section to the KASCADE-Grande $N_{c h}$ data to test how well the high-energy hadronic interaction model EPOSLHC describes the evolution of this observable with zenith angle. The result is presented in Table 1 along with the estimation from experimental data. In addition, predictions from the QGSJETII-02, QGSJETII-04, EPOS 1.99, SIBYLL 2.1 high-energy hadronic interaction models, as calculated in reference $[23]^{3}$, are also shown. In all cases, the values for $\Lambda_{c h}$ are presented with their total errors. The corresponding systematic errors have the same contributions as the errors estimated for $\Lambda_{\mu}$, but with the difference that in the case of $\Lambda_{c h}$ there is no influence from any correction function as the charged number of particles is measured with a better precision than $N_{\mu}$.

The results for the predictions of the attenuation length of $N_{c h}$ present an overall agreement with the experimental

\footnotetext{
${ }^{3}$ The same selection cuts that we employed in this study are also applied on these data. For the latter the available statistics is higher than for EPOS-LHC.
} 
value as observed in Table 1. Differences between the models and the measurement go from $0.25 \sigma$ (EPOS-LHC) up to $0.99 \sigma$ (SIBYLL 2.1). Here, again, from all hadronic interaction models, EPOS-LHC predicts the closer $\Lambda_{c h}$ value to the experimental value. This is a significant improvement of EPOS-LHC over the EPOS 1.99 model.

\section{Conclusions}

We have tested the predictions of the EPOS-LHC model regarding the zenith-angle evolution of the $N_{\mu}$ and $N_{c h}$ observables in the atmosphere by studying the corresponding attenuation lengths. The calculations were confronted with the measurements of the KASCADEGrande experiment. Preliminary studies suggest that predictions from EPOS-LHC have a better agreement with the experimental values than pre-LHC highenergy interaction models (QGSJETII-02, EPOS 1.99 and SIBYLL 2.1) and even the post-LHC model QGSJETII-04. However, in all cases, it is observed that the MC models do not satisfactorily predict the muon data, as they give lower muon attenuation lengths than the one measured with the KASCADE-Grande observatory. For the case of $\Lambda_{c h}$, MC predictions seem to be in better agreement with the measured data. For the case of EPOS-LHC, the preliminary result shows the lowest difference with the experimental result.

The authors would like to thank the members of the engineering and technical staff of the KASCADE-Grande Collaboration, who contributed to the success of the experiment. The KASCADEGrande experiment is supported by the BMBF and the Helmholtz Alliance for Astroparticle Physics - HAP of Germany, the MIUR and INAF of Italy, the Polish Ministry of Science and Higher Education and the Romanian Authority for Scientific Research UEFISCDI (PNII-IDEI grants 271/2011 and 17/2011). J.C.A.V. acknowledges the partial support of CONACyT and the
Coordinación de la Investigación Científica de la Universidad Michoacana.

\section{References}

[1] S.S. Ostapchenko, PRD 83, 014018 (2011)

[2] T. Pierog et al., arxiv: 1306.0121 (2013)

[3] T. Pierog, Proc. of the 23rd ECRS, J. of Physics: Conference Series 409, 012008 (2013)

[4] Y. Itow et al., EPJ Web of Conf. 49, 11001 (2013)

[5] P. Gunnellini et al., arxiv:1304.2943v1 (2013)

[6] G. Antchev et al. arxiv:1308.6722v1 (2013); The TOTEM Coll., EPL 96, 21002 (2011)

[7] M.Unger et al., EPJ Web of Conf. 52, 01009 (2013)

[8] T. Pierog, EPJ Web of Conf. 52, 03001 (2013)

[9] M. Bertaina et al., Poc. of the 33rd ICRC, ID0196

[10] A. Aab et al., arXiv:1307.5059 (2013)

[11] W.D. Apel et al., NIM A 620, 202 (2010)

[12] T. Antoni et al., NIM A 513, 490 (2003)

[13] P. Doll et al., NIM A 488, 517 (2002)

[14] S.D. Wick, C.D. Dermer and A. Atoyan, Astropart. Phys. 21, 125 (2004); J.R. Hoerandel, Astropart. Phys. 21 (2004) 241-265; V.S. Ptuskin et al., Astron. Astrophys. 268, 726 (1993); B. Peters, Il Nuo. Cim. 22, 800 (1961)

[15] A. De Rújula, Nucl. Phys. B, Proc. Suppl. 151, 23 (2005)

[16] T. Antoni et al., Astropart. Phys. 24, 1 (2005)

[17] W.D. Apel et al., PRL 107, 171104 (2011)

[18] W.D. Apel et al., PRD 87, 081101(R) (2013)

[19] W.D. Apel et al., Astropart. Phys. 47, 54 (2013)

[20] W.D. Apel et al., Adv. in Space Res. 53, 1456 (2014)

[21] G. Toma et al., these proceedings

[22] P. Luczak et al., these proceedings

[23] J.C. Arteaga-Velázquez et al., arxiv:1308.3202

[24] L. Cazon et al., arXiv:1405.5095 (2014)

[25] W.D. Apel et al., Astropart. Phys. 36, 183 (2012) 